\title{
Evaluation of Tranche in Securitization and Long-range Ising Model
}

\author{
K Kitsukawa $\dagger \dagger$, S Mori $\ddagger \dagger$ and M Hisakado $\Phi \S$ \\ $\dagger$ Graduate School of Media and Governance, Keio University, Endo 5322, Fujisawa, \\ Kanagawa 252-8520, Japan \\ $\ddagger$ Department of Physics, School of Science, Kitasato University, Kitasato 1-15-1 , \\ Sagamihara, Kanagawa 228-8555, Japan \\ ๑ Standard \& Poor's, Marunouchi 1-6-5, Chiyoda-ku, Tokyo 100-0005, Japan
}

\begin{abstract}
This econophysics work studies the long-range Ising model of a finite system with $N$ spins and the exchange interaction $\frac{J}{N}$ and the external field $H$ as a model for homogeneous credit portfolio of assets with default probability $P_{d}$ and default correlation $\rho_{d}$. Based on the discussion on the $(J, H)$ phase diagram, we develop a perturbative calculation method for the model and obtain explicit expressions for $P_{d}, \rho_{d}$ and the normalization factor $Z$ in terms of the model parameters $N$ and $J, H$. The effect of the default correlation $\rho_{d}$ on the probabilities $P\left(N_{d}, \rho_{d}\right)$ for $N_{d}$ defaults and on the cumulative distribution function $D\left(i, \rho_{d}\right)$ are discussed. The latter means the average loss rate of the "tranche" (layered structure ) of the securities (e.g. CDO), which are synthesized from a pool of many assets. We show that the expected loss rate of the subordinated tranche decreases with $\rho_{d}$ and that of the senior tranche increases linearly, which are important in their pricing and ratings.
\end{abstract}

PACS numbers: 05.50.+q,02.50.-r

Submitted to: Physica A

$\dagger$ kj198276@sfc.keio.ac.jp

$\ddagger$ mori@sci.kitasato-u.ac.jp

$\S$ masato_hisakado@standardpoors.com 


\section{Introduction}

The statistical properties of the models for credit risks have been widely discussed in the past ten years from the standpoint of financial engineering [1, 2] and econophysics [3. 4]. In the context of econophysics, the mechanism of systemic failure in banking has been studied [5, 6]. Power law behavior of the distributions of avalanches and several scaling laws in the context of percolation theory were found. On the other hand, in financial engineering, the evaluation of the effect of the correlation $\rho$ between the rates of return of assets or between the default of assets is a hot topic and is widely discussed from theoretical and empirical viewpoints. Empirically, historically realized values of correlations and their implied values, which are estimated based on the market value of credit derivatives, are compared and their discrepancies, called correlation risk premium, attract investors' interests from the viewpoint of portfolio management [7]. Theoretically, many statistical models are proposed for modeling credit risk of the pool of many assets [2, 8, 9, 10, 11, 12, 13, 14, 15, 16]. There are two categories in the models. The models in the first category use two state discrete variables which describe whether the asset is defaulted or not 9, 10, 5, 11. In the financial literature a two-valued variable $x_{i}(i=1, \cdots, N)$ takes values 0 and 1 depending on whether the $i$-th asset described by $x_{i}$ is not defaulted or defaulted. The default probability $P_{d}$ is defined by the average number of defaulted assets $N_{d}$ per an asset as $P_{d}=\frac{\left\langle N_{d}\right\rangle}{N}$. Here $<>$ means the expectation value. Ising Spin variable $S= \pm 1$ is also used and it is related to $x$ as $S=1-2 x$. Moody's Binomial (Expansion) approach 9], Moody's correlated Binomial model [10], Long-range Ising model [1] are in this category. The default correlation is defined by the simultaneous default probability. If we denote the probability distribution of two asset $i, j$ as $P_{i j}\left(x_{i}, x_{j}\right)$, the default correlation $\rho_{d}$ is defined by

$$
\rho_{d}=\frac{P_{i j}(1,1)-P_{i}(1) \times P_{j}(1)}{\sqrt{P_{i}(1)\left(1-P_{i}(1)\right)} \sqrt{P_{j}(1)\left(1-P_{j}(1)\right)}} .
$$

Here, $P_{i}\left(x_{i}\right)$ and $P_{j}\left(x_{j}\right)$ are the probability distributions of $x_{i}$ and $x_{j}$ and they are calculated from the joint probability distribution $P_{i j}\left(x_{i}, x_{j}\right)$.

In the second category, the models adopt a continuous variable for the earning rate of an asset and correlation between the earning rates is introduced [8, 12, 13, 14,. On the assumption that the earing rates obey multivariate normal distribution with correlation $\rho_{a}$, the probability $P_{i, j}$ for the simultaneous default of the $i$-th and $j$-th assets is given by

$$
P_{i, j}=\frac{1}{2 \pi \sqrt{1-\rho_{a}^{2}}} \int_{-\infty}^{\Phi^{-1}\left(P_{i}\right)} \int_{-\infty}^{\Phi^{-1}\left(P_{j}\right)} \exp \left(-\frac{u^{2}-2 \rho_{a} u v+v^{2}}{2\left(1-\rho_{a}^{2}\right)}\right) d u d v
$$

Here $P_{i}$ and $P_{j}$ are the default probabilities of the $i$-th and $j$-th assets and $\Phi^{-1}(x)$ is the inverse function of the normal distribution function. The variables $u, v$ mean the earing rates of the two assets. If the random variable $u$ (or $v$ ) becomes lower than $\Phi^{-1}\left(P_{i}\right)$ (resp. $\left.\Phi^{-1}\left(P_{j}\right)\right)$, the $i$-th $(j$-th) asset is judged to be defaulted. The correlation parameter $\rho_{a}$ is named as "asset correlation" and $\rho_{a}$ and $\rho_{d}$ are related via the equation 
(11). The conditionally independent model [12, such as the Merton based model, the credit metrix model [13] and the copula model [14] are in the second category.

The reason why default or asset correlations are widely discussed recently is that the pricing of Asset backed Security (ABS), like CDO, needs detailed information about the probabilities $P\left(N_{d}\right)$ for $N_{d}$ defaults. Here CDO is an abbreviation for Collateralised debt obligation, which is a financial innovation to securitise portfolios of defaultable assets. The portfolio of the underlying debts (assets) collateralizes the securitites (obligations), $\mathrm{CDO}$ is a kind of ABS. Securitization by CDO, we mean to synthesize securities based on a pool of many assets, like loans (CLO), commercial bonds (CBO) etc. In the process, layered structure is introduced and securities with high priority (reliability), which is called senior tranche, and those with low priority (called subordinated tranche or equity) are synthesized. Between the senior tranche and the equity, the mezzanine tranche with middle priority is also synthesized. The difference between them is that if some of the assets in the pool are defaulted, the security with lower priority loses its value at first. If the rate of defaulted assets $\frac{N_{d}}{N}$ exceeds some threshold value $P_{c}$, e.g. $P_{c}=7 \%$ for the mezzanie tranche and $P_{c}=10 \%$ for the senior tranche, those with higher priority begin to lose their values. The equity play the role of "shock absorber". By the "tranche" structure, the risk of the senior tranche is reduced and investors feel safe about the investment. On the other hand, the interest rates of the securities of the equity and the mezzanine tranche are set to be higher than those of the senior tranche and the subordinated tranches are high-risk-high-return products.

The default correlation becomes important when one try to estimate the expected loss in each tranche, which is essential in the evaluation of its price (premium). For example, we assume a pool of $N$ homogeneous assets with default probability $P_{d}$. If there is no correlation between the defaults, $P\left(N_{d}\right)$ is the binomial distribution $B\left(N, P_{d}\right)$ and has a peak at $N_{d}=P_{d} N$. The standard deviation is $\sqrt{N P_{d}\left(1-P_{d}\right)} \simeq \sqrt{N P_{d}}$ for small $P_{d}$. If the threshold value $P_{c}$ is large enough, the upper tranche does not suffer from the defaults in the pool. On the other hand, in the extreme case where the default correlation is $\rho_{d}=1$, all assets behave in the same way and there are only two cases. One case is that all assets are not defaulted and the probability for the case is $1-P_{d}$. The other case is that all assets are defaulted simultaneously and the probability is $P_{d}$. In the strong correlation limit $\left(\rho_{d}=1\right)$, when there occur defaults, all assets become defaulted simultaneously. Both senior and subordinated tranches lose their values completely. If there occurs no default, both tranche does not suffer from any damage. The essential problem is to know the $\rho_{d}$ dependence of the probabilities $P\left(N_{d}\right)$. It is important to estimate the expected loss rate of each tranche based on $P\left(N_{d}\right)$. In addition, we should also study which probabilistic model is good or useful in order to describe the behaviors of the assets.

This paper deals with these problems. The organization of the paper is as follows. In section 2, we study the $(J, H)$ phase diagram of finite size long-range Ising model and show that the assets begin to be correlated in the "Two Peak" Phase in the $(J, H)$ plane. The realistic magnitude of the default correlation ranges from $1 \%$ to several $\%$ 
[2], only the Two Peak Phase is interesting from the financial engineering viewpoint. Section 3 is devoted to the calculation of the important parameter $P_{d}$ and $\rho_{d}$ in terms of $J, H$ and $N$. Here, we develop a perturbation method which is based on the discussions in section 2. Up to zero-th order in the perturbation theory, $P\left(N_{d}\right)$ is expressed as the superposition of two binomial distributions, corresponding to the two peaks of $P\left(N_{d}\right)$. The developed method and obtained relations are useful when one apply the long-range Ising model to the evaluation and hedging of the securities with tranches. In section 4 , we study the $\rho_{d}$ dependence of $P\left(N_{d}, \rho_{d}\right)$ and of the expected loss rates of the tranches. For the latter purpose, we introduce the cumulative distribution $D\left(i, \rho_{d}\right)$ and discuss that they are directly related with the average loss rates of tranches. As the correlation becomes strong (with fixed default probability $P_{d}$ ), the left peak becomes taller and moves towards to the origin $\left(N_{d}=0\right)$. The right peak also becomes taller and shifts to $N_{d}=N$. Its area approaches to $P_{d}$ as $\rho_{d}$ comes close to 1 . These behaviors are different from those of the binomial expansion approach, where $P\left(N_{d}\right)$ has only one peak and its shape becomes broader as $\rho_{d}$ increases. We then discuss the $\rho_{d}$ dependence of $D\left(i, \rho_{d}\right) . \quad D(i)$ for large $i$ increases linearly with $\rho_{d}$ and the senior tranche cannot avoid the default damage of the assets pool, even when we set $P_{c}$ to be large. This crucial behavior of the long-range Ising model has been pointed out previously [11, we have clarified the importance in the evaluation of the tranches. Section 5 is dedicated to concluding remarks and future problems. We discuss the usefulness of the long-range Ising model from the viewpoint of financial engineering.

\section{Model and Phase Diagram in $(J, H)$ plane}

We use Ising Spin variables $S_{1}, S_{2}, \cdots, S_{N}= \pm 1$ which represent states of assets in the reference pool. Here $S_{i}=-1$ indicates default of $i$-th asset and $S_{i}=1$ means that the $i$-th asset is not defaulted. We denote the number of $S= \pm 1$ spins by $N_{ \pm}$, so the number of defaulted assets $N_{d}$ is $N_{-}$. The probability distribution for the states of the assets is assumed to be described by the following canonical distribution with the long-range Ising model of a finite system with $N$ spins and the exchange interaction $\frac{J}{N}$ and the external filed $H$, which are measured in units of Boltzmann constant times temperature.

$$
P\left(S_{1}, S_{2}, \cdots, S_{N}\right)=\frac{1}{Z_{N}(J, H)} \exp \left(\frac{J}{2 N} \sum_{1 \leq i, j \leq N} S_{i} S_{j}+H \sum_{i=1}^{N} S_{i}\right) .
$$

We do not omit the $i=j$ terms in the Hamiltonian for later convenience. As is wellknown, the exchange interaction $-\frac{J}{N} S_{i} S_{j}$ controls the strength of the correlation between $S_{i}$ and $S_{j}$ and the external field $H$ favors one of the two spin states. In the actual case where the spin variable represents the states of the assets, the default probability $P_{d}$ is at most a few percent and almost all assets are not defaulted $(S=1)$. The sign of the external field $H$ is set to be $H>0$.

The reason to choose the long-range Ising model is that it gives the default distribution $P\left(N_{d}\right)$ directly. In [11], another motivation for the long-range Ising model 
has been discussed and their conclusion is that the model is the most natural choice from the viewpoint of the Maximum Statistical Entropy principle. The two parameters $J$ and $H$ are introduced as Lagrange multipliers which ensure that the default probability and the default correlation of the model are $p_{d}$ and $\rho_{d}$. From the economical viewpoint, we can interpret the model as a kind of factor model. Here, the term 'factor' means the systematic risk factor or the state of the business cycle [2]. In a boom, we have fewer defaults than in a recession. We denote the state of the business cycle as $H^{\prime}$ and assume that the defaults of the assets are independent from each other, conditional on the realization of the systematic factor $H^{\prime}$. The joint probabilities for the assets $S_{1}, S_{2}, \cdots, S_{N}$ and the business cycle variable $H^{\prime}$ is assumed to be written as

$$
P_{\text {factor }}\left(S_{1}, S_{2}, \cdots, S_{N}, H^{\prime}\right)=\frac{1}{Z_{N}(J)} \exp \left(H^{\prime} \sum_{i=1}^{N} S_{i}\right) \times P\left(H^{\prime}\right) .
$$

Here, the random variable $H^{\prime}$ obeys the probability density function $P\left(H^{\prime}\right)$ and the denominator $Z_{N}(J)$ is the normalization term. Condition on the realization $H^{\prime}=H$, the each asset state becomes independent from each other and the default probability $P_{d}$ is given as

$$
P_{d}=\operatorname{Prob}\left(S_{i}=-1\right)=\frac{e^{-2 H}}{1+e^{-2 H}} .
$$

The default probability $P_{d}$ is a decreasing function of $H$ and $H$ for a boom (recession) is large (small). In order to derive the long-range Ising model starting from the above factor mode, we assume that $H^{\prime}$ obeys the standard normal distribution with mean $H$ and variance $J / N$.

$$
P\left(H^{\prime}\right)=\frac{1}{\sqrt{2 \pi J}} \exp \left(-\frac{\left(H^{\prime}-H\right)^{2}}{2 J / N}\right)
$$

By averaging over the possible realization of $H^{\prime}$ weighted with the above $P\left(H^{\prime}\right)$, we obtain the expression for the long-range Ising model.

$$
\begin{aligned}
& \int_{-\infty}^{\infty} P_{\text {factor }}\left(S_{1}, S_{2}, \cdots, S_{N}, H^{\prime}\right) d H^{\prime}=\int_{-\infty}^{\infty} \frac{1}{Z_{N}(J)} \exp \left(H^{\prime} \sum_{i=1}^{N} S_{i}\right) \times P\left(H^{\prime}\right) d H^{\prime} \\
& =\frac{1}{Z_{N}(J, H)} \exp \left(\frac{J}{2 N} \sum_{1 \leq i, j \leq N} S_{i} S_{j}+H \sum_{i=1}^{N} S_{i}\right)
\end{aligned}
$$

The validity of the Maximum Statistical Entropy principle or the factor model with the normally distributed business factor $H^{\prime}$ should be checked by the comparison with other more reliable models.

The Hamiltonian of the long-range Ising model depends on the spin variables only through the combination of the magnetization $M=\sum_{i=1}^{N} S_{i}$. There is a simple relation between $N_{-}=N_{d}$ and $M$ as $M=N_{+}-N_{-}=N-2 N_{d}$, the default number distribution function $P\left(N_{d}\right)$ is

$$
P\left(N_{d}\right)=\frac{\exp \left(\frac{J}{2 N} N^{2}+H N\right)}{Z_{N}(J, H)}{ }_{N} C_{N_{d}} \exp \left(\frac{2 J}{N} N_{d}^{2}-(2 J+2 H) N_{d}\right) .
$$




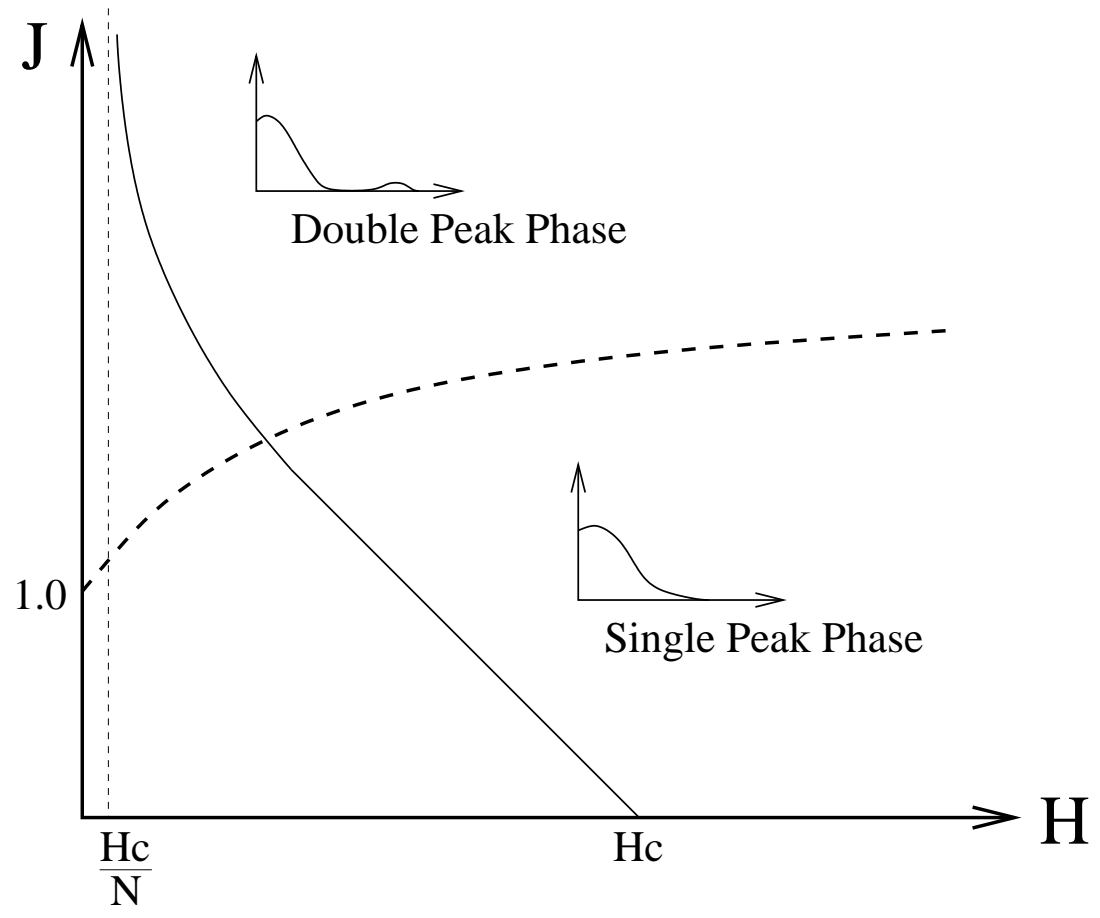

Figure 1. Phase diagram in $(J, H)$ plane. For large $H$ and small $J, P\left(N_{d}\right)$ has a single peak at $N_{d} \simeq P_{d} N$. We call the region as "Single Peak" Phase. For small $H$ and large $J$, there are two peaks in $P\left(N_{d}\right)$ and we call the region "Two Peak" Phase. The phase boundary is depicted with the broken line $(---)$. The solid line $(-)$ corresponds to a constant $P_{d}$ line. It starts at $(J, H)=\left(0, H_{C}\right)$, where $\rho_{d}=0$. In the $J \rightarrow \infty$ limit, the line approaches $H=\frac{H_{c}}{N}$ asymptotically and $\rho_{d} \rightarrow 1$.

The default probability $P_{d}$ is defined by the expectation value of $N_{d}$ as

$$
P_{d} \equiv \frac{<N_{d}>}{N}
$$

Here $<>$ is the expectation value with the probability distribution (8) . For $J=0$, the probability distribution (8) becomes that of the binomial distribution $B\left(N, P_{d}\right)$ and there is a relation between $H$ and $P_{d}$ as

$$
H=-\frac{1}{2} \log \left(\frac{P_{d}}{1-P_{d}}\right) \text { for } \quad J=0 .
$$

We denote this value of $H$ as $H_{C}$. On the other hand, for $J \rightarrow+\infty$ limit, there are only 2 configurations with all spins up or all spins down that have nonzero probabilities. The probabilities are

$$
P(1,1, \cdots, 1)=\frac{1}{1+e^{-2 H N}} \quad P(-1,-1, \cdots,-1)=\frac{e^{-2 H N}}{1+e^{-2 H N}} .
$$

From the relation $P(-1,-1, \cdots,-1)=P_{d}$, one obtains the following relation between $H$ and $P_{d}$ for $J=\infty$ as

$$
H_{J=\infty}=\frac{H_{C}}{N} .
$$


For general $J, H$ and $N$, it is difficult to obtain $P_{d}$. However, for large enough $N$, by changing variable from $N_{-}$to $n_{-}=\frac{N_{-}}{N}$ in eq. (8) , we can estimate $P_{d}=<n_{-}>$by the saddle point approximation. The saddle point equation is

$$
n_{-}=\frac{e^{4 J n_{-}-(2 J+2 H)}}{e^{4 J n_{-}-(2 J+2 H)}+1} .
$$

Of course, by changing variable from $n_{-}$to the magnetization per spin $m=1-2 n_{-}$, the saddle point equation is transformed into the famous self-consistent equation of the magnetization $m=\tanh (J m+H)$ [17]. Depending on the values of the parameters $J, H(>0)$, there are two cases. For large $H$ and small $J$, the equation (12) has only one solution $n_{-*}$. We call this region in the $(J, H)$ plain as "One Peak" Phase, because the probability distribution $P\left(N_{d}\right)$ has a single peak at $N n_{-*} . P_{d}$ is almost the same with $n_{-*}$ in the One Peak Phase. For small $H$ and large $J$, the equation (12) has three solutions, two are at maxima $n_{-1 *}<n_{-2 *}$ and one is at minimum. We call the region in the $(J, H)$ plane as "Two Peak" Phase, as the reader may easily anticipate the reason. In the case, there is no simple relation between $P_{d}$ and the solutions $n_{-1 *}, n_{-* 2}$. If $H>0$ is large, the solution $n_{-1 *}$ is almost the same with $P_{d}$. However, when the correlation $\rho_{d}$ is large, the strength of $H$ is of the order of $\mathrm{O}\left(\frac{1}{N}\right)$ and we cannot neglect the second peak $n_{-* 2}$. In the case, $n_{-1 *}<P_{d}$ and the average value of $n_{-1 *}$ and $n_{-2 *}$ with $P\left(n_{-* 1}\right)$ and $P\left(n_{-* 2}\right)$ corresponds to the value of $P_{d}$. For example, when $\rho_{d}=1$ and $J=\infty$, the average value of $n_{-1 *}=0$ and $n_{-2 *}=1$ with probabilities eq.(111) is equal to $P_{d}$. In figure 1, we summarize the situation. The solid curve $(-)$ in the $(J, H)$ plane corresponds to the constant $P_{d}$ line. The dotted line $(---)$ is the "phase transition" line between the One-Peak Phase and the Two Peak Phase. In the remainder of the section, we study the correlation $\rho_{d}$ in the $(J, H)$ phase diagram. We will see that $\rho_{d}$ is almost zero in the One Peak Phase. Only in the Two Peak Phase $\rho_{d}$ can take nonzero value.

We discuss the default correlation $\rho_{d}$ and recall the definition (11). In order to obtain $P\left(S_{1}, S_{2}\right)$, in equation (3), we take the trace over $S_{3}, S_{4}, \cdots, S_{N}$.

$$
P\left(S_{1}, S_{2}\right)=\prod_{j=3}^{N} \sum_{S_{j}= \pm 1} P\left(S_{1}, S_{2}, \cdots, S_{N}\right) .
$$

The trace over $S_{3}, \cdots, S_{N}$ is replaced by the summation over $N_{-}$of $N^{\prime \prime}=N-2$ Spins. We obtain

$$
\begin{aligned}
& P\left(S_{1}, S_{2}\right)=\frac{1}{Z_{N}(J, H)} \exp \left(\frac{J}{N} S_{1} S_{2}+H\left(S_{1}+S_{2}\right)\right) \times \\
& \sum_{N_{-}=0}^{N^{\prime \prime}} N^{\prime \prime} C_{N_{-}} e^{\left(\frac{J}{N}\left(S_{1}+S_{2}\right)\left(N^{\prime \prime}-2 N_{-}\right)+\frac{J}{N}\left(N^{\prime \prime}-2 N_{-}\right)^{2}+H\left(N^{\prime \prime}-2 N_{-}\right)\right)} .
\end{aligned}
$$

If the system size $N$ is large, the summation over $N_{-}$is replaced by the estimation at the saddle points. In the One-Peak region, the saddle point is at $n_{-*}=P_{d}$ and $P\left(S_{1}, S_{2}\right)$ is given by

$$
P\left(S_{1}, S_{2}\right) \propto \exp \left(\frac{J}{N} S_{1} S_{2}+H\left(S_{1}+S_{2}\right)\right) \exp \left(-J\left(S_{1}+S_{2}\right) \times 2 n_{-*}\right) .
$$




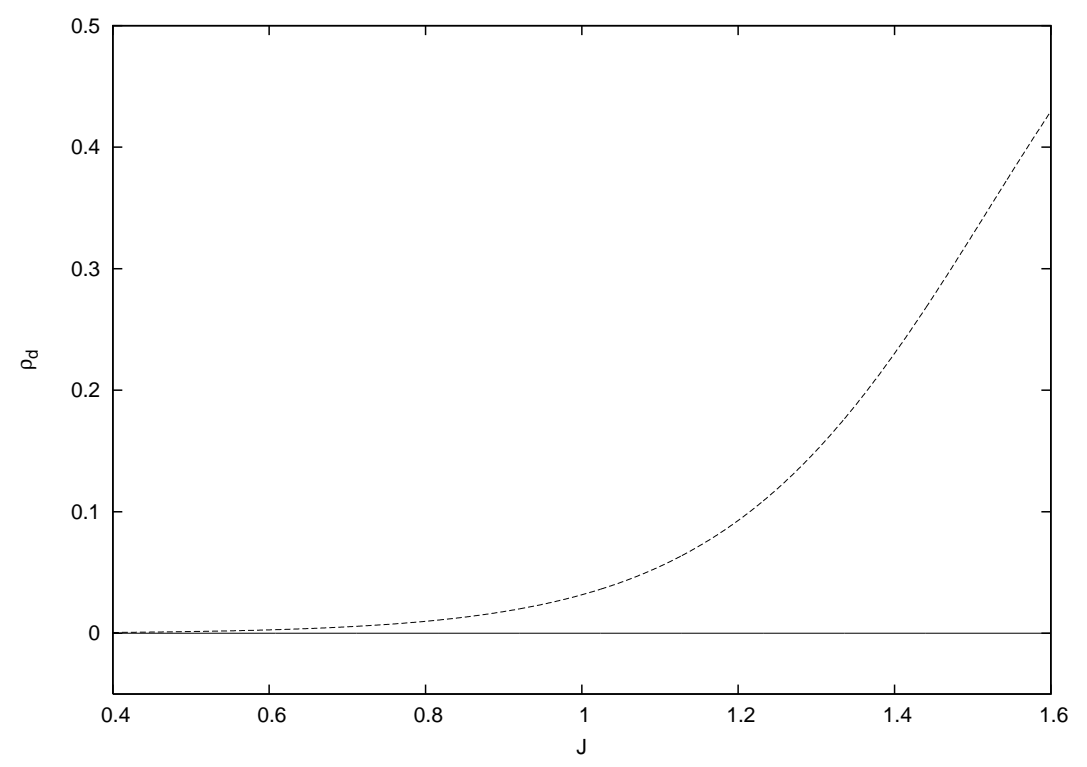

Figure 2. Second peak contribution in $\rho_{d}$. Along the line with eq.(17), we plot the approximated estimation for $\rho_{d}$. The lower solid line $(-)$ shows the data from eq.(15) and the data from eq.(16) are depicted with upper dotted curve $(\cdots \cdots)$.

In the Two Peak region, two saddle points contribute to the summation and $P\left(S_{1}, S_{2}\right)$ is estimated as

$$
\begin{aligned}
& P\left(S_{1}, S_{2}\right) \propto \exp \left(\frac{J}{N} S_{1} S_{2}+H\left(S_{1}+S_{2}\right)\right) \times \\
& {\left[e^{-J\left(S_{1}+S_{2}\right) \times 2 n_{-1 *}} \times P\left(n_{-1 *}\right)+e^{-J\left(S_{1}+S_{2}\right) \times 2 n_{-2 *}} \times P\left(n_{-2 *}\right) .\right]}
\end{aligned}
$$

Here $P\left(n_{-1 *}\right)$ and $P\left(n_{-2 *}\right)$ are the probabilities for the two peaks $n_{-1 *}$ and $n_{-2 *}$. In the One Peak phase, the constant $P_{d}$ line in the $(J, H)$ plane is almost given by the following relation between $J$ and $H$

$$
H=\tanh ^{-1}\left(1-2 P_{d}\right)-J\left(1-2 P_{d}\right) .
$$

We calculate the default correlation $\rho_{d}$ with equations (15) and (16) on the above approximate constant $P_{d}$ line. About the two saddle points $n_{-1 *}, n_{-2 *}$ and their probabilities $P\left(n_{-1 *}\right), P(n-2 *)$, we take them the values at $J=\infty$ and $\rho_{d}=1$. We set $n_{-1 *}=0, n_{-2 *}=1$ and $P\left(n_{-1 *}\right)=1-P_{d}, P\left(n_{-2 *}\right)=P_{d}$. We set $P_{d}=0.01$ and we plot $\rho_{d}$ vs $J$ in figure 2. We see that the correlation with equation (15), which is plotted with solid line $(-)$, does not become large even in the Two Peak Region. On the other hand, $\rho_{d}$ with equation (16), which is depicted with dotted line $(\cdots \cdots)$ becomes large in the Two Peak Region. We see that the existence of the second peak in $P\left(N_{d}\right)$ plays a crucial role in the emergence of correlation in the long-range Ising model.

\section{Perturbative Calculation and Second Peak Contribution}

In this section, we try to calculate several quantities of interest of the long-range Ising model. In particular, we obtain the expressions for $P_{d}$ and $\rho_{d}$ in terms of the model 
parameter $J, H$ and $N$. In addition, we also obtain the expression for the probability (or weight) of the second peak $P_{\text {all }}$, which means that almost all assets are defaulted [11. The probability $P_{\text {all }}$ plays a crucial role when one discuss the evaluation of the tranche.

When one calculate $P_{d}, \rho_{d}$, one way is to calculate $\left\langle S_{i}>\right.$ and $\left\langle S_{i} S_{j}>\right.$. Here, we calculate the moment of $N_{d}$ with the probability distribution eq.(8). The default probability is then given by $P_{d}=\frac{\leq N_{d}>}{N}$. About the default correlation $\rho_{d}$, we start from the following relation.

$$
\rho_{d}=\frac{P(-1,-1)-P(-1)^{2}}{P(-1)(1-P(-1))}=\frac{<S_{1} S_{2}>-<S_{1}><S_{2}>}{\left(1-<S_{1}>\right)\left(1+<S_{1}>\right)}
$$

The magnetization $M=N<S_{1}>$ and $N_{d}$ is related as $M=N-2 N_{d}$ and $N(N-1)<S_{1} S_{2}>=<M^{2}>-N$, we obtain the following expression

$$
\rho_{d}=\frac{\sigma_{N_{d}}^{2}+\frac{1}{N-1}\left(<N_{d}^{2}>-N<N_{d}>\right)}{<N_{d}>\left(N-<N_{d}>\right)} .
$$

In order to calculate the moment $\left\langle N_{d}^{l}>\right.$ with eq.(8) , the quadratic term $\exp \left(\frac{2 J}{N} N_{d}^{2}\right)$ prevents us from taking summation over $N_{d}$. As we have noted previously, the distribution with $J=0$ is binomial distribution and taking summation over $N_{d}$ is easy. In addition, the $P_{d}$ is at most a few percent and the distribution $P\left(N_{d}\right)$ have a peak very close to $N_{d}=0$ (and the second peak at $N_{d} \simeq N$ in the Two Peak Phase). We expand the quadratic term as

$$
\exp \left(\frac{2 J}{N} N_{d}^{2}\right)=\sum_{k=0}^{\infty} \frac{1}{k !}\left(\frac{2 J}{N} N_{d}^{2}\right)^{k} .
$$

and perform the calculation of the moment $\left\langle N_{d}^{l}>\right.$ perturbatively. The expansion is about $\frac{2 J}{N} N_{d}^{2}$, which is evaluated as $\frac{2 J}{N} N_{d}^{2} \simeq 2 J N P_{d}^{2}$. In the actual risk portfolio problem, $P_{d}$ is at most $2 \sim 3 \%$ and the system size $N$ is several hundred, the perturbative approximation is considered to be applicable. We also note that, in the Two Peak Phase, the above expansion should be carried out also at $N_{d}=N$.

In order to perform the calculation in more concrete manner, we use variables $N_{ \pm}$ and start from the following expression for the Hamiltonian.

$$
\begin{aligned}
-\mathcal{H} & =\frac{J}{2 N} M^{2}+H M \\
& =\frac{J}{2 N} N^{2}+H N-(2 H+2 J) N_{-}+\frac{2 J}{N} N_{-}^{2} \\
& =\frac{J}{2 N} N^{2}-H N-(2 J-2 H) N_{+}+\frac{2 J}{N} N_{+}^{2} .
\end{aligned}
$$

In the vicinity of $N_{-}=0$, we denote $P\left(N_{-}\right)$as $P_{-}\left(N_{-}\right)$and we can expand the quadratic term in eq.(21). Likewise, in the vicinity of $N_{-}=N\left(N_{+}=0\right)$, we call $P\left(N_{-}\right)$as $P_{+}\left(N_{-}\right)$ and it can also be expanded in $N_{+}$.

$$
\begin{aligned}
P_{-}\left(N_{-}\right) & =\frac{1}{Z}{ }_{N} C_{N_{-}} e^{H N} e^{-(2 H+2 J) N_{-}+\frac{2 J}{N} N_{-}^{2}} \\
& =\frac{1}{Z}{ }_{N} C_{N_{-}} e^{H N} e^{-(2 H+2 J) N_{-}} \times \sum_{k=0}^{\infty} \frac{1}{k !}\left(\frac{2 J}{N} N_{-}^{2}\right)^{k}
\end{aligned}
$$




$$
\begin{aligned}
P_{+}\left(N_{-}\right) & =\frac{1}{Z}{ }_{N} C_{N-N_{-}} e^{-H N} e^{-(2 J-2 H)\left(N-N_{-}\right)+\frac{2 J}{N}\left(N-N_{-}\right)^{2}} \\
& =\frac{1}{Z}{ }_{N} C_{N-N_{-}} e^{-H N} e^{-(2 J-2 H)\left(N-N_{-}\right)} \times \sum_{k=0}^{\infty} \frac{1}{k !}\left(\frac{2 J}{N}\left(N-N_{-}\right)^{2}\right)^{k} .
\end{aligned}
$$

$Z$ is the normalization constant to ensure that $\sum_{N_{-}=0}^{N_{1}} P\left(N_{-}\right)=1$. To the zero-th order perturbation approximation $P_{-}\left(N_{-}\right)$and $P_{+}\left(N_{-}\right)$are binomial distributions and $P\left(N_{-}\right)$ is given by the superposition of these distributions. We summarize the situation as

$$
P\left(N_{-}\right)= \begin{cases}P_{-}\left(N_{-}\right) & \left(0 \leq N_{-} \leq L\right) \\ P_{+}\left(N_{-}\right) & \left(0 \leq N-N_{-}<N-L\right) .\end{cases}
$$

Here $L$ is set to be at the middle of the interval $[0, N]$.

The moment $\left\langle N_{-}^{l}\right\rangle$ is calculated with the following equation.

$$
<N_{-}^{l}>=\sum_{N_{-}=0}^{N} P\left(N_{-}\right) N_{-}^{l}=\sum_{N_{-}=0}^{L} P_{-}\left(N_{-}\right) N_{-}^{l}+\sum_{N_{-}=L}^{N} P_{+}\left(N_{-}\right) N_{-}^{l} .
$$

The summation over $N_{-}$is from 0 to $L$, however $P_{-}\left(N_{-}\right)$damps rapidly in $N_{-}$, it is not so bad to change the range from $[0, L]$ to $[0, N]$. About $P_{+}\left(N_{-}\right)$the range of $N_{-}$ is $[L, N]$. We change variable from $N_{-}$to $N_{+}=N-N_{-}$and denote the probability distribution $P_{+}\left(N_{-}=N-N_{+}\right)$also as $P_{+}\left(N_{+}\right)$.

$$
P_{+}\left(N_{+}\right)=\frac{1}{Z}{ }_{N} C_{N_{+}} e^{-H N} e^{-(2 J-2 H)\left(N_{+}\right)+\frac{2 J}{N}\left(N_{+}\right)^{2}} .
$$

$P_{+}\left(N_{+}\right)$also damps rapidly in $N_{+}$, we will change the summation range from $[0, N-L)$ to $[0, N] .<N_{-}^{l}>$ is then calculated perturbatively as

$$
\begin{aligned}
& <N_{-}^{l}>=\sum_{N_{-}=0}^{N} P_{-}\left(N_{-}\right) N_{-}^{l}+\sum_{N_{+}=0}^{N} P_{+}\left(N_{+}\right)\left(N-N_{+}\right)^{l} \\
& =\frac{1}{Z} e^{H N} \sum_{N_{-}=0}^{N}{ }_{N} C_{N_{-}} e^{-(2 H+2 J) N_{-}} \sum_{k=0}^{\infty} \frac{1}{k !}\left(\frac{2 J}{N} N_{-}^{2}\right)^{k} N_{-}^{l} \\
& +\frac{1}{Z} e^{-H N} \sum_{N_{+}=0}^{N}{ }_{N} C_{N_{+}} e^{-(2 J-2 H) N_{+}} \sum_{k=0}^{\infty} \frac{1}{k !}\left(\frac{2 J}{N} N_{+}^{2}\right)^{k}\left(N-N_{+}\right)^{l} .
\end{aligned}
$$

The normalization constant $Z$ is calculated as

$$
\begin{aligned}
Z & =e^{H N} \sum_{N_{-}=0}^{N}{ }_{N} C_{N_{-}} e^{-(2 H+2 J) N_{-}} \sum_{k=0}^{\infty} \frac{1}{k !}\left(\frac{2 J}{N} N_{-}^{2}\right)^{k} \\
& +e^{-H N} \sum_{N_{+}=0}^{N}{ }_{N} C_{N_{+}} e^{-(2 J-2 H) N_{+}} \sum_{k=0}^{\infty} \frac{1}{k !}\left(\frac{2 J}{N} N_{+}^{2}\right)^{k} \\
& =Z_{-}+Z_{+} .
\end{aligned}
$$

In equation (29), we denote the two terms as $Z_{ \pm}$, which come from the summation over $N_{-}$and $N_{+}$.

In the above calculation, moments of the binomial distribution appears frequently. We introduce the following unnormalized binomial moments $\left[N_{ \pm}^{l}\right]$.

$$
\left[N_{ \pm}^{l}\right]=\sum_{N_{ \pm}=0}^{N}{ }_{N} C_{N_{ \pm}} e^{\beta_{ \pm} N_{ \pm}} N_{ \pm}^{l}
$$




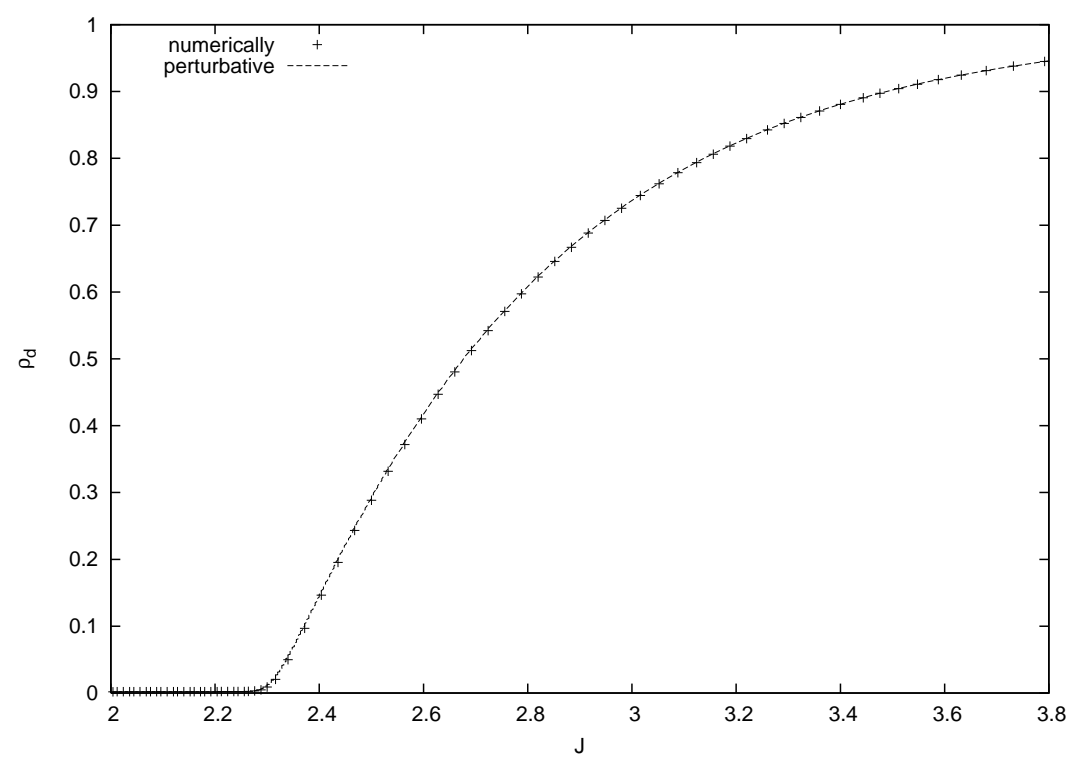

Figure 3. The default correlation $\rho_{d}$ on a constant $P_{d}$ line. $P_{d}=0.01$ and we plot $\rho_{d}$ versus $J . H$ is set to be the value which realize $P_{d}=0.01$ for $N=50$. The solid line ( - ) shows the result from the perturbative calculation up to second order in $\frac{2 J}{N}$ and the line with + symbols shows numerically calculated exact data.

The parameters $\beta_{ \pm}$are defined as $\beta_{ \pm}=-2(J \mp H)$. Calculations of $\left[N_{ \pm}^{l}\right]$ is straightforward. The zero-th moment $\left[1_{ \pm}\right]$is given by

$$
\left[1_{ \pm}\right]=\left(1+e^{\beta_{ \pm}}\right)^{N} .
$$

The $l$-th moment $\left[N_{ \pm}^{l}\right]$ is then obtained by differentiating $\left[1_{ \pm}\right]$with respect to $\beta_{ \pm}$ repeatedly.

$$
\left[N_{ \pm}^{l}\right]=\frac{\partial^{l}}{\partial \beta_{ \pm}^{l}}\left(1+e^{\beta_{ \pm}}\right)^{N} .
$$

We show the results for the first 6 moments, which are necessary for the second order perturbative calculation.

$$
\begin{aligned}
{\left[N_{ \pm}^{1}\right] } & =\left(1+e^{\beta_{ \pm}}\right)^{N}\left(N_{1} x_{ \pm}\right) \\
{\left[N_{ \pm}^{2}\right] } & =\left(1+e^{\beta_{ \pm}}\right)^{N}\left(N_{1} x_{ \pm}+N_{2} x_{ \pm}^{2}\right) \\
{\left[N_{ \pm}^{3}\right] } & =\left(1+e^{\beta_{ \pm}}\right)^{N}\left(N_{1} x_{ \pm}+3 N_{2} x_{ \pm}^{2}+N_{3} x_{ \pm}^{3}\right) \\
{\left[N_{ \pm}^{4}\right] } & =\left(1+e^{\beta_{ \pm}}\right)^{N}\left(N_{1} x_{ \pm}+7 N_{2} x_{ \pm}^{2}+6 N_{3} x_{ \pm}^{3}+N_{4} x_{ \pm}^{4}\right) \\
{\left[N_{ \pm}^{5}\right] } & =\left(1+e^{\beta_{ \pm}}\right)^{N}\left(N_{1} x_{ \pm}+15 N_{2} x_{ \pm}^{2}+25 N_{3} x_{ \pm}^{3}+10 N_{4} x_{ \pm}^{4}+N_{5} x_{ \pm}^{5}\right) \\
{\left[N_{ \pm}^{6}\right] } & =\left(1+e^{\beta_{ \pm}}\right)^{N}\left(N_{1} x_{ \pm}+31 N_{2} x_{ \pm}^{2}\right. \\
& \left.\quad+90 N_{3} x_{ \pm}^{3}+65 N_{4} x_{ \pm}^{4}+15 N_{5} x_{ \pm}^{5}+N_{6} x_{ \pm}^{6}\right)
\end{aligned}
$$


where $N_{i}=\frac{N !}{(N-i) !}$ and $x_{ \pm}=\frac{e^{\beta_{ \pm}}}{1+e^{\beta_{ \pm}}}$. In general, the $l$-th binomial moment $\left[N_{ \pm}^{l}\right]$ is calculated as

$$
\left[N_{ \pm}^{l}\right]=\left(1+e^{\beta_{ \pm}}\right)^{N} \sum_{k=1}^{l} y_{ \pm k}^{l} N_{k} x_{ \pm}^{k},
$$

where the coefficients $y_{ \pm k}^{l}$ for $N_{k} x_{ \pm}^{k}$ is calculated with the following recursive relations.

$$
y_{ \pm k}^{l}=y_{ \pm k-1}^{l-1}+k y_{ \pm k}^{l-1}
$$

and with the conditions $y_{ \pm k}^{l}=0$ for $k>l$ and $y_{1}^{1}=1$.

With these preparations, we are ready to write down the results. The perturbative calculation of the normalization constant $Z$ is given as

$$
\begin{aligned}
Z & =Z_{-}+Z_{+} \\
& =e^{H N} \sum_{k=0}^{\infty} \frac{1}{k !}\left(\frac{2 J}{N}\right)^{k}\left[N_{-}^{2 k}\right]+e^{-H N} \sum_{k=0}^{\infty} \frac{1}{k !}\left(\frac{2 J}{N}\right)^{k}\left[N_{+}^{2 k}\right] .
\end{aligned}
$$

The moment $<N_{-}^{l}>$ is given by

$$
\begin{aligned}
& <N_{-}^{l}>=\frac{1}{Z} e^{H N} \sum_{k=0}^{\infty} \frac{1}{k !}\left(\frac{2 J}{N}\right)^{k}\left[N_{-}^{2 k+l}\right] \\
& +\frac{1}{Z} e^{-H N} \sum_{k=0}^{\infty} \frac{1}{k !}\left(\frac{2 J}{N}\right)^{k} \sum_{m=0}^{l}{ }_{l} C_{m}(-1)^{m} N^{l-m}\left[N_{+}^{2 k+m}\right] .
\end{aligned}
$$

Putting these results into $P_{d}=\frac{\left\langle N_{d}>\right.}{N}$ and eq.(19), the expressions for $P_{d}$ and $\rho_{d}$ in terms of model parameters $N, J, H$ are obtained. In addition, the weight of the second peak $P_{\text {all }}$, that is the probability of almost all assets are defaulted, is estimated as

$$
P_{\text {all }}=\frac{Z_{+}}{Z} \text {. }
$$

As we have noted previously, the zero-th order approximation means that we express $P\left(N_{d}\right)$ as a superposition of two binomial distributions. In the case, the results for $Z$ and $P_{d}, \rho_{d}$ can be written down in the following simple expressions.

$$
\begin{aligned}
Z^{0} & =e^{H N}\left(1+e^{\beta_{-}}\right)^{N}\left(1+\gamma^{N}\right) \\
P_{d}^{0} & =\frac{x_{-}+\gamma^{N}\left(1-x_{+}\right)}{1+\gamma^{N}} \\
\rho_{d}^{0} & =\frac{1}{P_{d}^{0}\left(1-P_{d}^{0}\right)} \frac{\gamma^{N}}{\left(1+\gamma^{N}\right)^{2}}\left(1-\left(x_{-}+x_{+}\right)\right)^{2} \\
\gamma & =e^{-2 H}\left(\frac{1+e^{\beta_{+}}}{1+e^{\beta_{-}}}\right)
\end{aligned}
$$

The subscript (0) indicates the zero-th order perturbation results. In figure 3, we shows the result for $\rho_{d}^{2}$ along the constant $P_{d}$ line. $P_{d}$ is set to be $P_{d}=0.01$ and with solid line $(-)$ we show the data from the above perturbative calculation up to second order in $\frac{2 J}{N}$. The line with + symbols depicts the numerical data. The two lines coincide well and the match is very good as long as $P_{d}$ is set to be small. Figure 4 is the 3 -dimensional plot of the data in $\left(J, H, \rho_{d}\right)$ space. $\rho_{d}$ begins to be large in the Two Peak region and its rapid growth is well captured by the above perturbative calculation. 


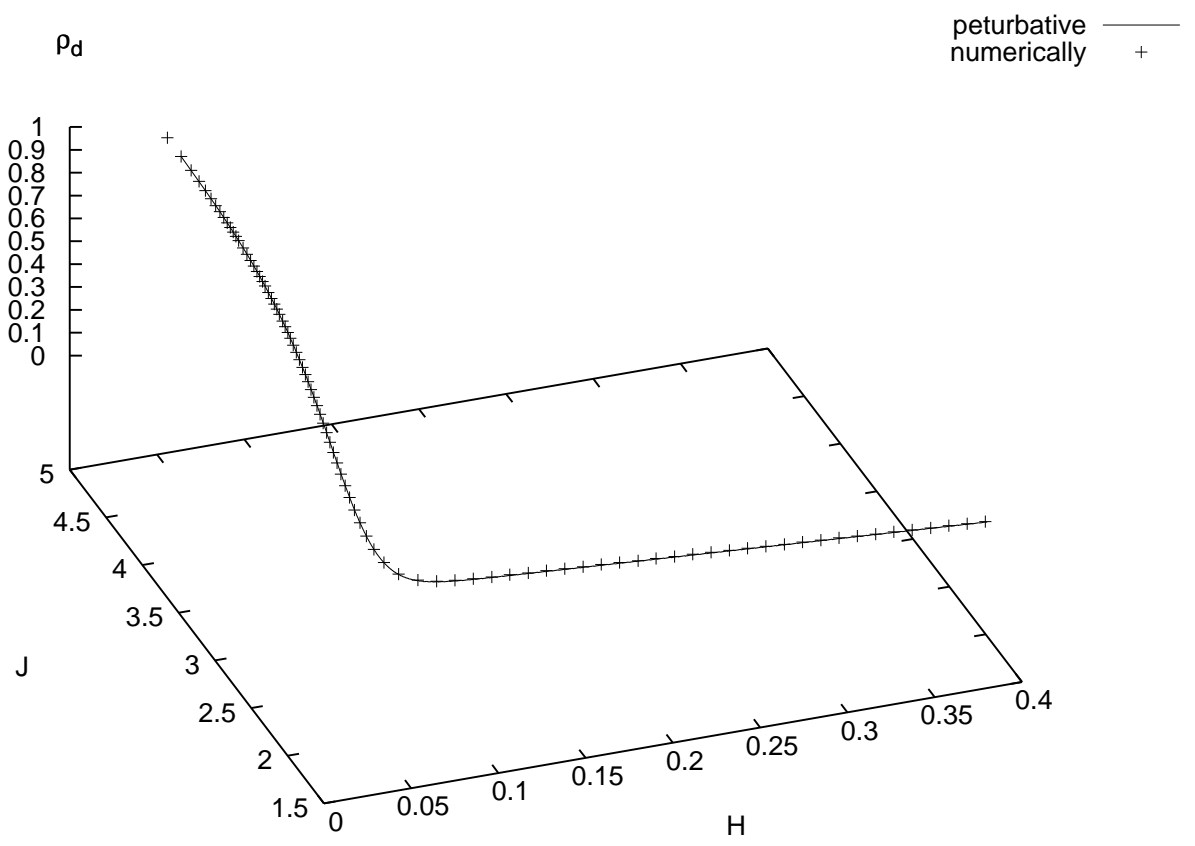

Figure 4. 3-dimensional plot of $\rho_{d}$ in $\left(J, H, \rho_{d}\right)$ space. The solid line $(-)$ shows the result from the perturbative calculation up to second order in $\frac{2 J}{N}$ and the line with + symbols depicts numerically calculated exact data. The conditions are the same with those in figure 3

\section{Effect of $\rho_{d}$ on $P\left(N_{d}\right)$ and on average loss rates of tranches}

We would like to discuss the effect of default correlation $\rho_{d}$ on the probabilities $P\left(N_{d}\right)$ and on the tranche synthesized from the pool of the homogeneous assets. In order to discuss the latter case, we introduce the cumulative distribution function $D\left(i, \rho_{d}\right)$, which is directly related with the average loss rate of the tranche. The default rate $P_{d}$ and the system size $N$ is fixed. When we show numerical data, we set $N=100$ and $P_{d}=0.05$.

At first, we discuss the former case, the effect on the probabilities $P\left(N_{d}, \rho_{d}\right)$. Here we write down their $\rho_{d}$ dependence explicitly. The $\rho_{d}=0$ case is easy and $P\left(N_{d}, 0\right)$ is only the binomial distribution $B\left(N, P_{d}\right)$. It has a single peak at $N P_{d}$ and the width is roughly $2 \sqrt{N P_{d}}$. For $N_{d}=0, P(0,0)=\left(1-P_{d}\right)^{N}$ and as $\rho_{d}$ becomes large, $P\left(0, \rho_{d}\right)$ approaches $1-P_{d}$. In figure 5 we plot $P\left(N_{d}, \rho_{d}\right)$ versus $\rho_{d}$ for $N_{d} \leq 10$. The system size $N=100$ and $P_{d}=0.05$. $P\left(0, \rho_{d}\right)$ grows monotonically as $\rho_{d}$ grows. For $1 \leq N_{d} \leq 5=N P_{d}, P\left(N_{d}, \rho_{d}\right)$ at first increases and then decreases as a function of $\rho_{d}$. On the other hand, for $N_{d} \geq 5=N P_{d}, P\left(N_{d}, \rho_{d}\right)$ decreases with $\rho_{d}$. $P_{d}$ is small and $P\left(N_{d}, 0\right)$ damps rapidly in $N_{d}$ for $N_{d} \geq 5, P\left(N_{d}, \rho_{d}\right)$ is almost zero for any $\rho_{d}$, which holds for $10<N_{d} \leq 90$. Figure [ 6 depicts the plots of $P\left(N_{d}, \rho_{d}\right)$ for $90 \leq N_{d} \leq 100 . P(N, 0)=P_{d}^{N} \simeq 0$ and $P(N, 1)=P_{d}, P\left(100, \rho_{d}\right)$ grows monotonically 


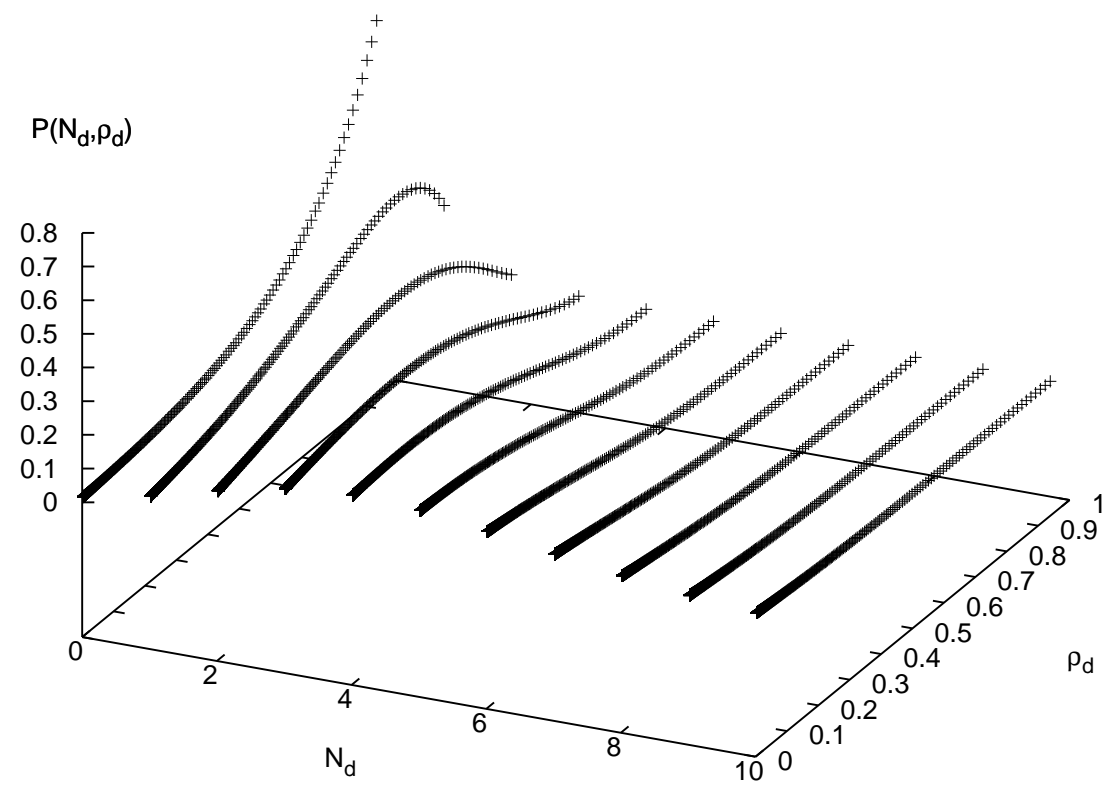

Figure 5. Plot of $P\left(N_{d}, \rho_{d}\right)$ vs $\rho_{d} .0 \leq N_{d} \leq 10$ and $N=100, P_{d}=0.05$.

from 0 to $P_{d}=0.05$. For $90 \leq N_{d} \leq 99, P\left(N_{d}, \rho_{d}\right)$ is upward convex with respect to $\rho_{d}$. The area of the second peak becomes greater with the increase of $\rho_{d}$ and $P\left(N_{d}, \rho_{d}\right)$ increases for $N_{d} \simeq N$. As $\rho_{d}$ becomes large, the width of the second peak becomes narrow and $P\left(N_{d}, \rho_{d}\right)$ for $N_{d} \neq N$ decreases. On the other hand, $P\left(N, \rho_{d}\right)$ increases monotonically to $P_{d}$.

To sum up, for $\rho_{d}=0, P\left(N_{d}, 0\right)$ is $B\left(N, P_{d}\right)$ and it has a single peak. The width of the peak is order $2 \sqrt{N P_{d}}$ and it is small for small $P_{d}$. As $\rho_{d}$ grows, the system is in the Two Peak Phase. At the zero-th order perturbative approximation, $P\left(N_{-}\right)=P_{-}\left(N_{-}\right)+P_{+}\left(N_{+}\right)$is a superposition of two binomial distributions. $P_{-}\left(N_{d}\right)$ has a peak at $N_{d} \leq N P_{d}$ and is approximately obeys $B\left(N, x_{-}\right)$. On the other hand, $P_{+}\left(N_{d}\right)$ is $B\left(N, 1-x_{+}\right)$and has a peak at $N_{d} \sim N$. The increase in $\rho_{d}$ accompanies the increase in $J$, however the change of $H$ is not so large and it decreases slightly (See Figure 1). The first peak position of $P\left(N_{d}\right)$, which is governed by $\beta_{-}=-2(J+H)$, moves towards to $N_{d}=0$ as $J$ increases. The first peak becomes narrower and higher with the left slide and only $P\left(0, \rho_{d}\right)$ grows monotonically. For $0<N_{d} \leq N P_{d}, P\left(N_{d}, \rho_{d}\right)$ is upward convex with respect to $\rho_{d}$. $P\left(N_{d}, \rho_{d}\right)$ for $N_{d}>N P_{d}$ damps with $\rho_{d}$ monotonically. On the other hand, the second peak position, which is governed by $\beta_{+}=-2(J-H)$, shifts towards to $N_{d}=N$. The area of the second peak, which is calculated as in eq. (43), approaches $P_{d}$ and the width becomes narrow. $P\left(N, \rho_{d}\right)$ increases monotonically to $P_{d}$ with $\rho_{d}$ and $P\left(N_{d}, \rho_{d}\right)$ near $N_{d}=N$ is upward convex.

We would like to discuss the above effect on the tranche of securities synthesized 


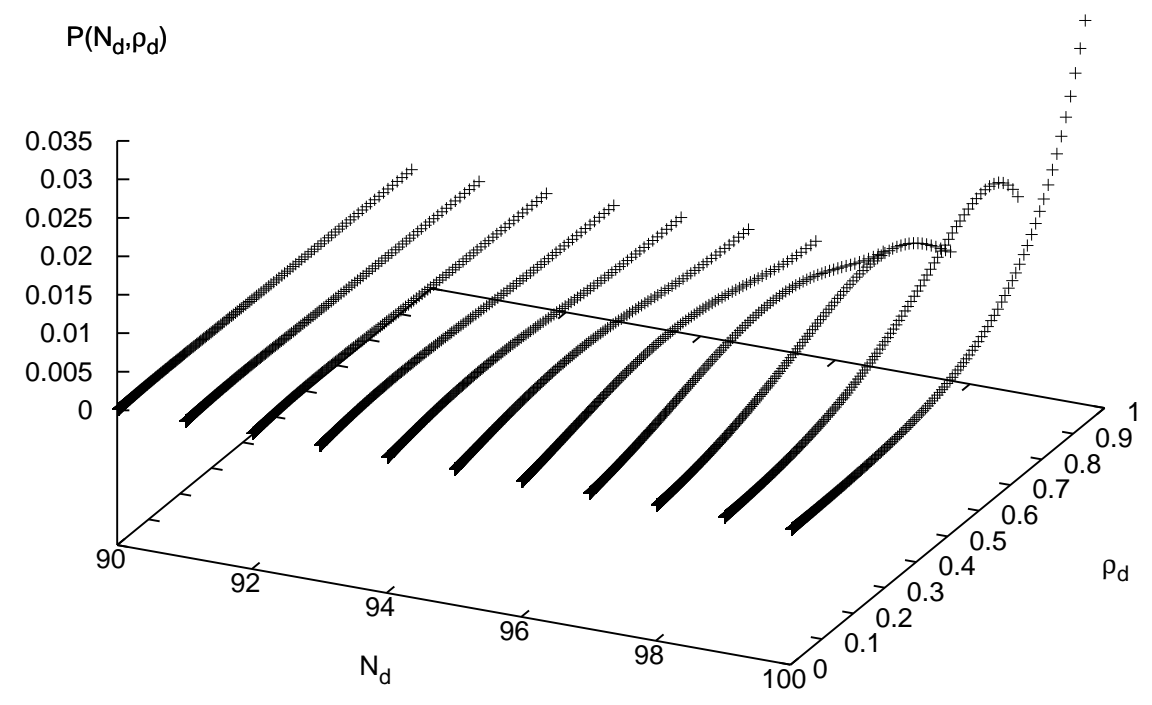

Figure 6. Plot of $P\left(N_{d}, \rho_{d}\right)$ vs $\rho_{d} .90 \leq N_{d} \leq 100$ and $N=100, P_{d}=0.05$.

from the homogeneous assets pool with parameters $P_{d}, \rho_{d}$. For the purpose, it is useful to introduce the cumulative distribution function $D(i, \rho)$, which is defined as

$$
D\left(i, \rho_{d}\right)=\sum_{N_{d}=i}^{N} P\left(N_{d}, \rho_{d}\right) \text {. }
$$

From the definition $D\left(0, \rho_{d}\right)=1$ and $D\left(1, \rho_{d}\right)=1-P\left(0, \rho_{d}\right)$ is the probability of the occurrence of default. We explain the relation between $D\left(i, \rho_{d}\right)$ and the evaluation of the tranche briefly.

The tranche for the interval $[i, j]$ implies that if the number of default $N_{d}$ is below $i\left(N_{d}<i\right)$, the tranche does not suffer from any damage. However, if $N_{d}$ exceeds or becomes equal to $i\left(N_{d} \geq i\right)$, it begins to lose its value. The value of the tranche is $\Delta=j-i+1$ in units of the number of assets (we assume that the values of all assets in the pool are equal.) and if defaults with $i \leq N_{d} \leq j$ occurs, it loses $\left(N_{d}-i+1\right)$ units. When $N_{d}$ exceeds $j\left(N_{d}>j\right)$, the tranche lose its value completely. The expected loss rate of the tranche $[i, j]$ is calculated as

$$
E(i \mid j)=\frac{1}{\Delta}\left(\sum_{k=i}^{j} P\left(k, \rho_{d}\right)(k-(i-1))+\Delta \sum_{k=j+1}^{N} P\left(k, \rho_{d}\right)\right) .
$$

The first terms comes from the partial damage in the tranche $\left(i \leq N_{d} \leq j\right)$ and the second term implies the contribution from its complete loss of the tranche $\left(N_{d} \geq j+1\right)$. $E(i \mid j)$ are directly related with the price of the tranche (premium), which can be 
observed in the market. For $j=i$, we denote $E(i \mid i)$ as $E(i)$ and call it as the expected loss rate at the $i$-th tranche. It is related with the cumulative distribution $D(i, \rho)$ as

$$
E(i)=E(i \mid i)=\sum_{k=i}^{N} P\left(k, \rho_{d}\right)=D\left(i, \rho_{d}\right) .
$$

$E(i)$ is useful, because we can reconstruct $E(i \mid j)$ as a sum of $E(k)$ as

$$
E(i \mid j)=\frac{1}{\Delta} \sum_{k=i}^{j} E(k) .
$$

The proof of the relation is straightforward.

$$
\begin{aligned}
\sum_{l=i}^{j} E(l) & =\sum_{l=i}^{j}\left(\sum_{k=l}^{N} P\left(k, \rho_{d}\right)\right)=\sum_{l=i}^{j}\left(\sum_{k=l}^{j} P\left(k, \rho_{d}\right)+\sum_{k=j+1}^{N} P\left(k, \rho_{d}\right)\right) \\
& =\sum_{k=i}^{j} P\left(k, \rho_{d}\right)(k-(i-1))+\Delta \sum_{k=j+1}^{N} P(k)=\Delta E(i \mid j)
\end{aligned}
$$

We note that, if we set $i=1$ and $j=N$ in equation (51), we obtain

$$
E(1 \mid N)=\frac{1}{N} \sum_{k=1}^{N} E(k)=P_{d} .
$$

Here we use the relation $E(1 \mid N)=P_{d}$, which is intuitively clear and can be proved as in the equation (52). From the second equality in eq. (153) that the average of the expected loss rate at each tranche is $P_{d}$, tranches look like to "share $P_{d}$ between them" or "toss $P_{d}$ to other tranches".

Now we discuss the effect of the default correlation $\rho_{d}$ on $E(i)=D\left(i, \rho_{d}\right)$. From the definition $D(i, \rho)=\sum_{k=i}^{N} P\left(k, \rho_{d}\right)$, we can understand the $\rho_{d}$ dependence easily from the previous discussions on $P\left(N_{d}, \rho_{d}\right)$. In figure [7] we show $D\left(i, \rho_{d}\right)$ for $91 \leq i \leq 100$. The area of the second peak increases monotonically to $P_{d}$ as we increase $\rho_{d}$, the cumulative distributions $D\left(i, \rho_{d}\right)$ also grow up to $P_{d} . P\left(N_{d}, \rho_{d}\right)$ is almost zero for $11 \leq N_{d} \leq 90$, $D\left(i, \rho_{d}\right)$ for $11 \leq i \leq 90$ behaves in the same way with these $D\left(i, \rho_{d}\right)$ for $i=91$. If $i$ becomes small, we see the contribution from the first peak in $P\left(N_{d}, \rho_{d}\right)$. For small $i$, the damps of $P\left(N_{d}, \rho_{d}\right)$ for $N_{d} \neq 0$ with respect to $\rho_{d}$ dominates the contribution from the second peak. $P\left(0, \rho_{d}\right)$ increases monotonically and $D\left(1, \rho_{d}\right)=1-P\left(0, \rho_{d}\right)$ decreases with $\rho_{d}$. $D\left(i, \rho_{d}\right)$ for $i \geq 2$ also decreases as in figure 8 , which shows $D\left(i, \rho_{d}\right)$ for $1 \leq i \leq 10$. These behaviors reflect the left shift and the width tinning of the first peak. For the intermediate value of $i$, the $\rho_{d}$ dependence of $D\left(i, \rho_{d}\right)$ is not monotonous. In figure 9, we depict $D\left(i, \rho_{d}\right)$ for $9 \leq i \leq 11$. Along with the shape change of the first peak with $\rho_{d}, D\left(i, \rho_{d}\right)$ at first decrease. Then, the contribution from the second peak dominates the decrease of the first peak contribution and $D\left(i, \rho_{d}\right)$ begins to increase. $D\left(i, \rho_{d}\right)$ is downward convex with respect to $\rho_{d}$ for the interval of $i$.

We note that the ranges where $D\left(i, \rho_{d}\right)$ is downward convex, $D\left(i, \rho_{d}\right)$ decreases monotonically, or $D\left(i, \rho_{d}\right)$ increases monotonically depends on the parameters $N, P_{d}$. The above discussions may not hold for other values of $N$ and $P_{d}$. In particular the range of the downward convex region, if we set $P_{d}=0.01$, we observe that it shift to 


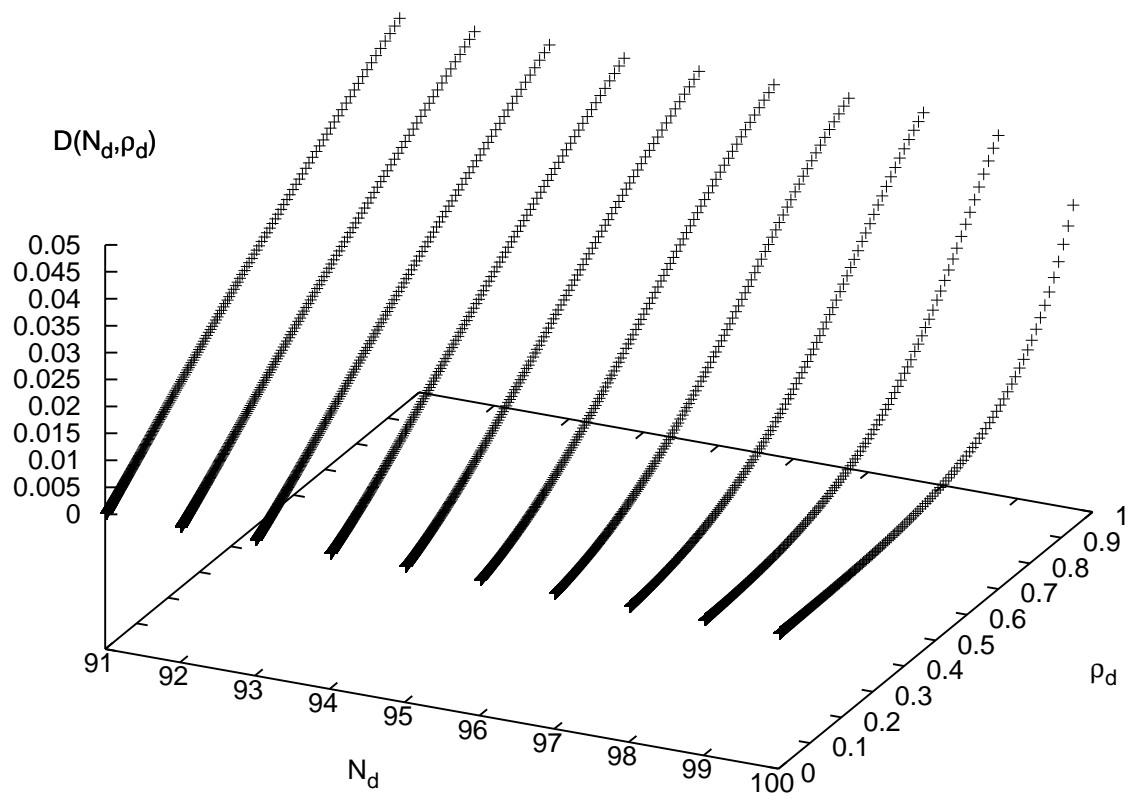

Figure 7. Plot of $D\left(i, \rho_{d}\right)$ vs $\rho_{d} .91 \leq i \leq 100$.

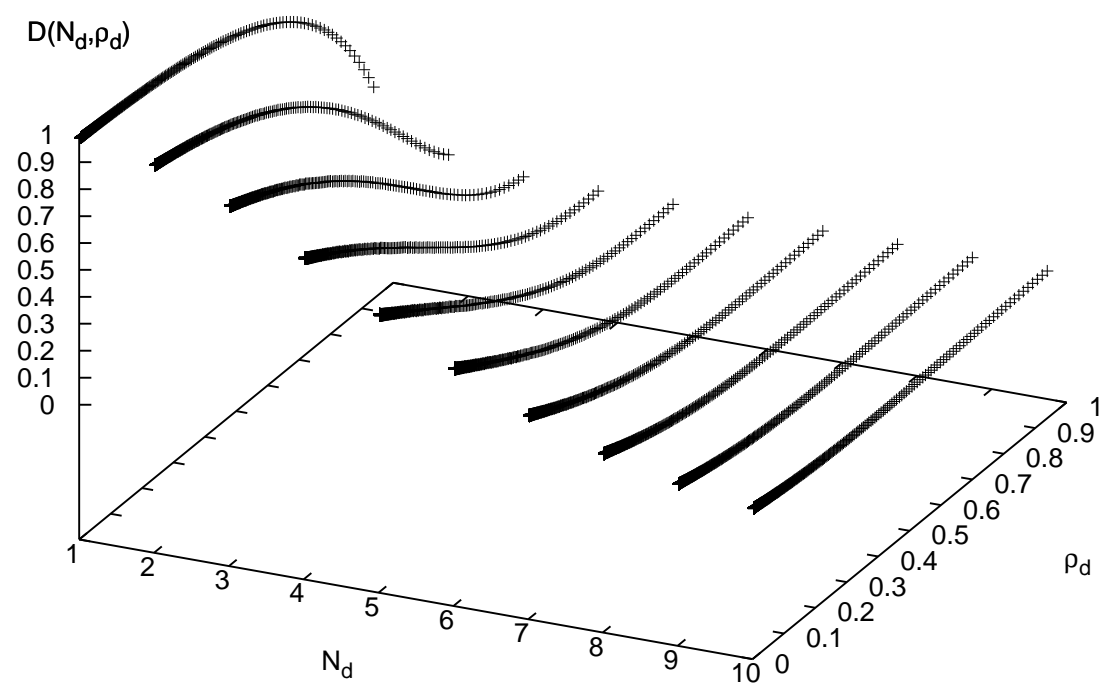

Figure 8. Plot of $D\left(i, \rho_{d}\right)$ vs $\rho_{d} . D\left(i, \rho_{d}\right)=\sum_{k=i}^{N} P\left(k, \rho_{d}\right)$ and $1 \leq i \leq 10$. 
the left. The positions of the boundaries between the regions are important from the viewpoints of risk management and rating of the securities, we should note this point.

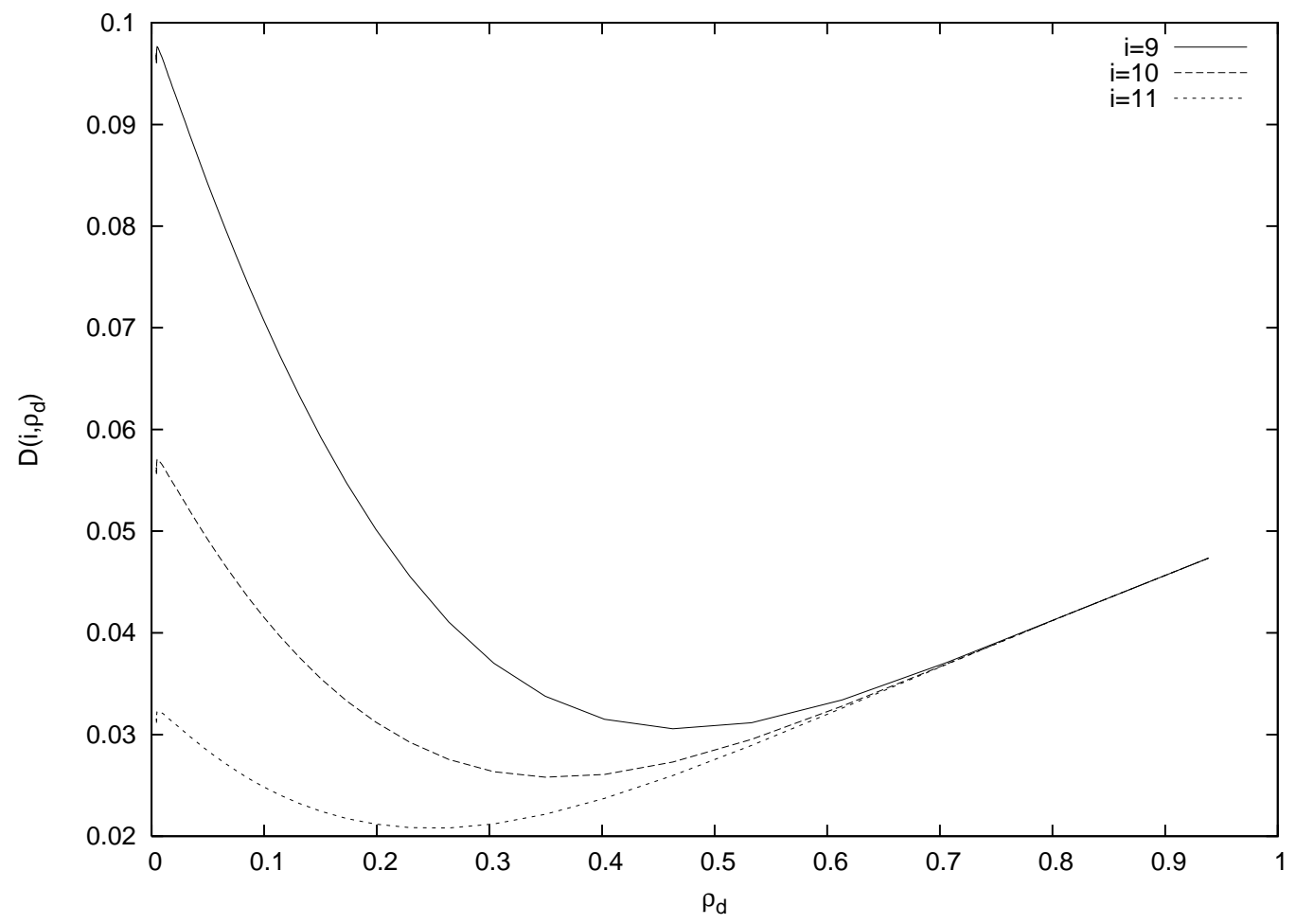

Figure 9. Plot of $D\left(i, \rho_{d}\right)$ vs $\rho_{d} .9 \leq i \leq 11$. Solid line $i=9$, broken line $i=10$ and dotted line $i=11$.

From these observations, we summarize the $\rho_{d}$ dependence of $E(i)=D\left(i, \rho_{d}\right)$.

- Senior tranche, the range $[i, j]$ with $i, j$ are set to be large, suffers from the default correlation seriously. $E(i)$ for the range increase linearly with $\rho_{d}$. It is approximately given by

$$
E(i)=\rho_{d} \times P_{d}
$$

If $\rho_{d}$ change from 0 to $1, E(i)$ change from 0 to $P d$ and the evaluations of the securities decrease almost linearly.

- Equity or subordinate tranche, the range $[i, j]$ is $[1, j]$ and $j$ is small. In the range $1 \leq k \leq j, D\left(k, \rho_{d}\right)$ damps monotonically with $\rho_{d}$ and $E(1 \mid j)$ also damps. The increase in $\rho_{d}$ causes the increase of the evaluation of the tranche.

- Mezzanine tranche, whose range is between the equity and the senior tranche. In the range, the behaviors of $D\left(k, \rho_{d}\right)$ depends on $P_{d}$ and the system size $N$. In the above mentioned $N=100, P_{d}=0.05$ case, $D\left(k, \rho_{d}\right)$ has downward convex shape for some intermediate values of $k$. 


\section{Concluding Remarks and Future Problems}

In this paper, we have studied the long-range Ising model as a model for a pool of $N$ homogeneous assets with default probability $P_{d}$ and default correlation $\rho_{d}$. We have studied in the $(J, H)$ plane, the behavior of $P_{d}$ and $\rho_{d}$. There are two phases in the $(J, H)$ plane. In the One Peak Phase, the probabilities $P\left(N_{d}\right)$ have a single peak at $N_{d} \simeq N P_{d}$. The correlation is almost zero in the phase. In the Two Peak Phase, there are two peaks in $P\left(N_{d}\right)$ and $\rho_{d}$ can take large value. The first peak is closer to origin than $N P_{d}$ and its area is larger than $1-P_{d}$. The second peak is at about $N$ and its area is less than $P_{d}$. The parameters $J, H$ should be chosen in the Two peak phase, if the model intends to describe the portfolio with some default correlation between the assets. We have developed the perturbative method and expressed $P\left(N_{d}\right)$ as a superposition of two binomial distributions with the above two peaks at zero-th order. We have obtained the closed form expression for $P_{d}, \rho_{d}$ and the weights for the second peak $P_{\text {all }}$, which means the probability that almost all assets are defaulted. These expressions are in good agreement with numerically calculated values and give an efficient method for the actual application of the long-range Ising model. Otherwise, for $P_{d}$ and $\rho_{d}$, it is difficult to know the parameters $J$ and $H$ and the long range Ising model is hard-to-use as a model for homogeneous credit risk portfolio.

Furthermore, we have studied the $\rho_{d}$ dependence of $P\left(N_{d}, \rho_{d}\right)$ and the cumulative distribution $D\left(i, \rho_{d}\right) . P\left(N_{d}, 0\right)$ is binomial distribution $B\left(N, P_{d}\right)$ and it has a peak at $N P_{d}$. As we increase $\rho_{d}$ from 0 to 1 , the profile of $P\left(N_{d}, \rho_{d}\right)$ changes from One peak shape to Two Peak shape. The first peak shifts to the left and its shape becomes higher and narrower. The second peak's area increases and it shifts to the right with the decrease of its width. At $\rho_{d}=1, P\left(N_{d}, 1\right)$ has two thin peaks at $N_{d}=0$ and $N_{d}=N$ and the probabilities are $P(0,1)=1-P_{d}$ and $P(N, 1)=P_{d}$. Other probabilities are zero. The cumulative distribution functions $D\left(i, \rho_{d}\right)$ correspond to the average loss rates of the $i$-th tranche. About the senior tranche, the range of the tranche $[i, j]$ is large. As $\rho_{d}$ increases, $D\left(i, \rho_{d}\right)$ increase almost linearly with $\rho_{d}$ like $D\left(i, \rho_{d}\right) \simeq \rho_{d} \times P_{d}$. The average loss rate of the senior tranche $[i, j]$ is given as a sum of $D\left(k, \rho_{d}\right)$ in the range $[i, j]$, the expectation value of the loss rate of the senior tranche also increases as $\rho_{d} \times P_{d}$. The price of the tranche is based on the average loss rate, the value of the senior tranche decreases with $\rho_{d}$. The range of the equity, the subordinated tranche, is near the origin and the $D\left(i, \rho_{d}\right)$ s decrease monotonically. The average loss rate of the equity decreases with $\rho_{d}$ and the price of the equity increase with $\rho_{d}$. The mezzanine tranche is between the equity and the senior tranche. The profile of $D\left(i, \rho_{d}\right)$ in the range depends on the model parameters $\rho_{d}, P_{d}$ and $N$. In the text example, $D\left(i, \rho_{d}\right)$ has a downward convex shape in some region. If the mezzanine range $[i, j]$ is chosen to lie in the region, the average loss rate also behaves similarly. However, other probabilistic model for a pool of assets, e.g. the copula model [14], suggest upward convex shape for the average loss of the mezzanine tranche. The discrepancy comes from the difference of the shapes of $P\left(N_{d}, \rho_{d}\right)$. The more complete comparison between the probabilistic models for a pool 
of correlated assets should be done.

As concluding remarks, we comment on the usage of the long-range Ising model and related future problems. As a statistical model for an ensemble of many assets, the long-range Ising model is an attractive one from the viewpoint of physicists. Its phase diagram and phase transitions are throughly studied and its analytic calculation method, like Hubbard-Stratanovich transformation, guides us how to make theoretically tractable models. On the other hand, from the viewpoint of financial engineers, the longrange Ising model is not so convenient. One reason is that the model parameters $J, H$ are not directly related with the observed data $P_{d}$ and $\rho_{d}$ (or $\rho_{a}$ ). Other statistical models incorporate these parameters as a model parameters. For example, the Moody's correlated binomial model gives $P\left(N_{d}\right)$ as a function of $P_{d}$ and $\rho_{d}$ explicitly. When one uses Ising model, it is necessary to know the parameters $J, H$ which correspond to $P_{d}, \rho_{d}$. The definition of $P_{d}$ and $\rho_{d}$ include the moments $\left\langle N_{-}^{l}>\right.$ or $\left\langle S_{i}>\right.$ and $\left\langle S_{i} S_{j}\right\rangle$, it is necessary to take the trace Tr. The long-range Ising model has the advantage that the trace $\operatorname{Tr}$ is reduced to the summation over the total magnetization $M=\sum_{j=1}^{N} S_{j}$ and the calculation is not so heavy task. Even so, this one step spoils the usefulness of the model. We have obtained a closed form expressions for $P_{d}$ and $\rho_{d}$ and try to circumvent the step. The computational time to obtain $J, H$ for given $P_{d}, \rho_{d}$ is reduced much and the failing of the model are partially overcome.

In order to apply the long-range Ising model to the evaluation of the tranche $[i . j]$ in more realistic situation, the assumption of homogeneity of the assets pool should be weakened. One step toward the direction is to introduce multi sectors and assume the homogeneity only in each sector. We label each sector by $I=1,2, \cdots K$ and $I$-th sector contains $N^{I}$ assets. In the $I$-th sector, the default rate is $P_{d}^{I}$ and the default correlation is $\rho_{d}^{I}$. Between different sectors, say between $I$-th and $J$-th sector, the default correlation is $\rho_{d}^{I J}$. We use Ising Spin variables $S_{j}^{I}$ to represent the states of the $j$-th asset in the $I$-th sector, the generalized long-range Ising model Hamiltonian for the probabilities $P\left(S_{j}^{I}\right)$ is

$$
-\mathcal{H}=\sum_{I=1}^{K} \frac{J^{I}}{2 N^{I}} M_{I}^{2}+H^{I} M_{I}+\sum_{1=I<J=K} \frac{J^{I J}}{\sqrt{N^{I} N^{J}}} M_{I} M_{J}
$$

As in the homogeneous model, the Hamiltonian depends on $S_{j}^{I}$ only through the magnetization of the $I$-th sector $M_{I}=\sum_{j=1}^{N^{I}} S_{j}^{I}$. When we set $\frac{J^{I}}{N^{I}}=\frac{J^{I J}}{\sqrt{N^{I} N^{J}}}=\frac{J}{N}$ and $H^{I}=H$, the model reduces to the homogeneous model with $N=\sum_{I=1}^{K} N^{I}, J, H$. The problem is to get the relation between $\rho_{d}^{I}, \rho_{d}^{I J}, P_{d}^{I}$ and $J^{I}, J^{I J}, H^{I}, N^{I}$. In order to accomplish the task, the phase diagram in $J^{I J}, J^{I}, H^{I}$ and the profile $P\left(N_{d}^{I}\right)$ should be cleared and it is left for future analysis. Furthermore, for more complex situation where $i$-th asset has default probability $P_{d}^{i}$ and the default correlation between $i$-th and $j$-th asset is $\rho_{d}^{i j}$, the model Hamiltonian becomes that of the random Ising spin systems. The exchange interaction $J_{i j}$ and the external field $H_{i}$ should be connected to $P_{d}^{i}$ and $\rho_{d}^{i j}$, which is also left for future problem. Other step is to discard the Ising model and adopt other probabilistic models. One possibility is the Moody's correlated binomial 
model, which uses two state variables $x=0,1$ for the state of an asset and incorporates $P_{d}$ and $\rho_{d}$ directly in the model parameters. Its generalization to the multi-sector case and more complex situations is an interesting problem. Other possibility is to introduce simplified version of the long-range Ising model. We use two state variable $x_{i}$ for the state of the $i$-th asset. The number of defaults $N_{d}$ is expressed as $N_{d}=\sum_{j=1}^{N} x_{j}$. The probabilities $P\left(x_{1}, x_{2}, \cdots, x_{N}\right)$ is given as

$$
P\left(x_{1}, x_{2}, \cdots, x_{N}\right)=(1-\alpha) p^{N_{d}}(1-p)^{N-N_{d}}+\alpha \delta_{N, N_{d}} .
$$

Instead of the superposition of two binomial distributions, we use $\alpha \delta_{N, N_{d}}$ for the second peak. The first peak is $B(N, p)$ and the parameters $\alpha, p$ are related with the default probability $P_{d}$ as $P_{d}=\alpha+(1-\alpha) p$. This probabilities $P\left(x_{1}, x_{2}, \cdots, x_{N}\right)$ is more tractable than the original probability distribution (3) and the generalizations to more complex situations may be carried out easily.

\section{Acknowledgments}

This work has received financial support from Kitasato University, project SCI:20051706.

\section{References}

[1] Fabozzi F J and Goodman L S 2001 Investing in Collateralized Debt Obligations (U.S. John Wiley \& Sons).

[2] Schonbucher P J 2003 Credit Derivatives Pricing Models : Model, Pricing and Implementation (U.S. John Wiley \& Sons) .

[3] Bouchaud J-P and Potters M 2000 Theory of Financial Risks(Cambridge University Press).

[4] Mantegna R N and Stanley H E 2000 An Introduction to Econophysics (Cambridge University Press).

[5] Aleksiejuk A and Holyst A 2001 Physica A299 198.

[6] Iori G 2001 Physica A299 205.

[7] Calamaro J P, Nassar T and Thakkar K 2004 Correlation: Trading Implications for Synthetic CDO Tranches (Deutsche Bank: Global Market Research, 27 September).

[8] Merton R 1974 The Journal of Finance 29449.

[9] Cifuettes A and O'Connor G 1996 The Binomial Expansion Method Applied to CBO/CLO Analysis (Moody's Investors Service).

[10] Witt G 2004 Moody's Correlated Binomial Default Distribution (Moody's Investors Service)August 10.

[11] Molins J and Vives E 2004 Long range Ising Model for credit risk modeling in homogeneous portfolios Preprint cond-mat/0401378

[12] Martin R, Thompson K and Browne C 2001 Risk July 86.

[13] Finger C C 2000 A Comparison of stochastic default rate models: Working Paper (The RiskMetrics Group).

[14] Li D X 2000 The Journal of Fixed Income 9(4)43.

[15] Duffie D and Gârleau 2001 Financial Analyst Journal 57(1)41-59.

[16] Duffie D and Singleton K J 2003 Credit Risk-Pricing, Measurement and Management (Princeton:Princeton University Press).

[17] Stanley H E 1983 Introduction to Phase Transitions and Critical Phenomena, vol 8 of International Series of Monographs on Physics (New York : Oxford University Press) . 